\title{
ON ADDITIVE K-THEORY WITH THE LODAY - QUILLEN *-PRODUCT
}

\author{
KATSUHIKO KURIBAYASHI and TOSHIHIRO YAMAGUCHI
}

\begin{abstract}
The *-product defined by Loday and Quillen [17] on the additive K-theory (the cyclic homology with shifted degrees) $K_{*}^{+}(A)$ for a commutative ring $A$ is naturally extended to a product (*-product) on the additive K-theory $K_{*}^{+}(\Omega)$ for a differential graded algebra $(\Omega, d)$ over a commutative ring. We prove that Connes' B-maps from the additive $\mathrm{K}$-theory $K_{*}^{+}(\Omega)$ to the negative cyclic homology $\mathrm{HC}_{*}^{-}(\Omega)$ and to the Hochschild homology $\mathrm{HH}_{*}(\Omega)$ are morphisms of algebras under the *-product on $K_{*}^{+}(\Omega)$. Applications to topology of Connes' B-maps are also described.
\end{abstract}

\section{$\S 0$. Introduction}

Let $A$ be an algebra over a commutative ring. Let $\operatorname{HC}_{n}^{-}(A)$ and $\mathrm{HH}_{n}(A)$ denote the negative cyclic homology and the Hochschild homology of $A$, respectively. In the algebraic K-theory, C. Hood and J. D. S. Jones [11] have constructed the Chern character $c h_{n}: K_{n}(A) \rightarrow \mathrm{HC}_{n}^{-}(A)$ which is a lift of the Dennis trace map Dtr: $K_{n}(A) \rightarrow \mathrm{HH}_{n}(A)$ by modifying basic construction due to Connes [5] and Karoubi [12]. When the algebra $A$ is commutative, the usual pairing of $K_{*}(A)$ and the product on $\mathrm{HC}_{*}^{-}(A)$ defined by Hood and Jones in [11] make the character $\mathrm{ch}_{*}$ into a morphism of algebras. In consequence, we can have the following commutative diagram in the category of graded algebras:

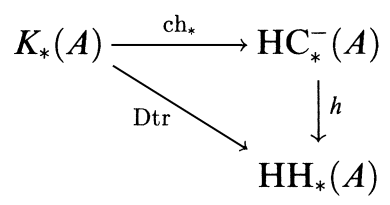

Here $h$ is the map induced from the natural projection to the Hochschild complex from the cyclic bar complex. The Chern character ch : $K_{0}(A) \rightarrow \mathrm{HC}_{0}^{-}(A)=\mathrm{HC}_{0}^{\mathrm{per}}(A)$ is connected with the ordinary Chern character $K(X) \rightarrow H_{\mathrm{de} \text { Rham }}^{\text {even }}(X ; \mathrm{C})$ when $A$ is the ring consisting of smooth

Received January 12, 1998. 
functions from a compact manifold $X$ to the complex number C (see, for example, [19, 6.2.9. Example]). Therefore, one may expect that the Chern character $\operatorname{ch}_{n}: K_{n}(A) \rightarrow \mathrm{HC}_{n}^{-}(A)$ or the Dennis trace map Dtr: $K_{n}(A) \rightarrow$ $\mathrm{HH}_{n}(A)$ becomes a map with value in the de $\mathrm{Rham}$ (singular) cohomology of some manifold (space) by replacing the algebra $A$ with an appropriate object concerning with the space.

Hochschild and (negative) cyclic homologies can be extended to functors defined on the category of commutative differential graded algebras (DGAs) over a commutative ring (see [8], [11], [4]). In particular, if we choose the de Rham complex $(\Omega(X), d)$ of a simply connected manifold $X$ as the DGA, the Hochschild and the negative cyclic homology of $\Omega(X)$ can be regarded as the real cohomology and the real T-equivariant cohomology of the space of free loops on $X$ respectively (see [8]), where T denotes the circle group. However, in algebraic K-theory, we can not expect such an extension. What is " $\mathrm{K}$ theory" which addmits an extension to a functor on the category of DGAs and in which there is a commutative diagram corresponding to (0.1)? We can consider the additive K-theory $K_{*}^{+}(A)$ (see [6]) as "K-theory", which is isomorphic to the positive cyclic homology group $\mathrm{HC}_{*-1}(A)$. Let $\phi$ be the isomorphism form $K_{*}^{+}(A)$ to $\mathrm{HC}_{*-1}(A)$ defined by Loday and Quillen in [17] and independently Tsygan in [21]. Tillmann's commutative diagram [20, Theorem 1] connects the dual of the Dennis trace map with the Connes Bmap by the dual of the isomorphism $\phi: K_{*}^{+}(A) \rightarrow \mathrm{HC}_{*-1}(A)$ when $A$ is a Banach algebra. Therefore it is natural to choose the Connes B-map $B_{\mathrm{HH}}: \mathrm{HC}_{*-1}(A) \rightarrow \mathrm{HH}_{*}(A)$ as a map in the additive $\mathrm{K}$-theory corresponding to the Dennis trace map in algebraic K-theory. The Connes' B-map $B_{\mathrm{HH}}: K_{*}^{+}(A) \cong \mathrm{HC}_{*-1}(A) \rightarrow \mathrm{HH}_{*}(A)$ has a natural lift $B$, which is also called Conne's B-map, to the negative cyclic homology $\mathrm{HC}_{*}^{-}(A)$. Moreover functors $\mathrm{HC}_{*}, \mathrm{HC}_{*}^{-}, \mathrm{HH}_{*}$ and the connecting maps can be extend on the category of DGAs by using the cyclic bar complex in [7] and [8]. In the consequence, we can obtain the following commutative diagram corresponding to $(0.1)$ in the category of graded modules:

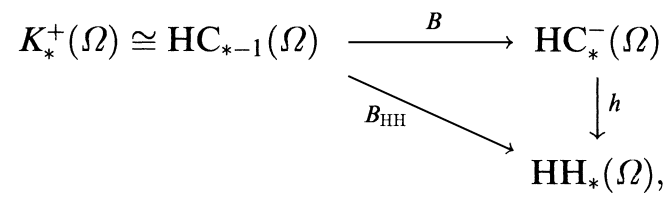

where $\Omega$ is a DGA. We propose a natural question that whether the diagram $(0.2)$ is commutative in the category of graded algebras, as well as the diagram $(0.1)$, under an appropriate product on $K_{*}^{+}(\Omega)$. To answer this question, we extend the *-product defined by Loday and Quillen [17] to a product on the additive K-theory (the cyclic homology with shifted degrees) of a 
DGA, which is an explicit version of that of Hood and Jones [11, Theorem 2.6]. Since the product is defined at chain level, we can see that

THEOREM 0.1. The diagram (0.2) is commutative in the category of graded algebras when the product of $K_{*}^{+}(\Omega)$ is given by the *-product.

Let $M$ be a simply connected manifold and $L M$ the space of all smooth maps from circle group T to $M$. By using the Connes' B-map $B_{\mathrm{HH}}$, we consider the vanishing problem of string class of a loop group bundle $L \operatorname{Spin}(n) \rightarrow L Q \rightarrow L M$. In the consequence, a generalization of the main theorem in [14] is obtained when the given manifold $M$ is formal (see Theorem 2.1).

We also show that the algebra structure of $\operatorname{HC}_{*}^{-}(\Omega)$ can be described with the *-product on $K_{*}^{+}(\Omega)$ via Connes' B-map $B: K_{*}^{+}(\Omega) \rightarrow \mathrm{HC}_{*}^{-}(\Omega)$ when the DGA $(\Omega, d)$ over a field $\mathbf{k}$ of characteristic zero is formal. This fact allows us to deduce the following theorem.

THeOREM 0.2. Let $X$ be a formal simply connected manifold. Then

$$
H_{\mathrm{T}}^{*}(L X ; \mathrm{R}) \cong\left\{H^{*}(L X ; \mathrm{R}) / \operatorname{Im}\left(B_{\mathrm{HH}} \circ I\right)\right\}^{*+1} \oplus \mathrm{R}[u]
$$

as an algebra, where $I: H^{*}(L X ; \mathrm{R})=\mathrm{HH}_{-*}(\Omega(X)) \rightarrow K_{-*}^{+}(\Omega(X))$ is the map in Connes' exact sequence $(1,1)$ mentioned in $\S 1$ for the de Rham complex $\Omega(X)$ with negative degrees and $\mathrm{R}[u]$ is the polynomial algebra over $u$ with degree 2. The multiplication of the algebra on the right hand side is given as follows; $w * u^{i}=0$ and $w * w^{\prime}=w \cdot B I w^{\prime}$, where is the cup product on $H^{*}(L X ; \mathbf{R})$. In particular,

(i) if $H^{*}(X ; \mathrm{R}) \cong \mathrm{R}[x] /\left(x^{s+1}\right)$ and $s>1$, then

$$
H_{\mathrm{T}}^{*}(L X ; \mathrm{R}) \cong \oplus_{k \geq 0,1 \leq j \leq s} \mathrm{R}\{\beta(j, k)\} \oplus \mathrm{R}[u]
$$

as an algebra, where $\operatorname{deg} \beta(j, k)=j \operatorname{deg} x+k((s+1) \operatorname{deg} x-2)-1$, $\beta(j, k) * \beta\left(j^{\prime}, k^{\prime}\right)=0$ and $\beta(j, k) * u=0$ for any $j, k, j^{\prime}, k^{\prime}$, and

(ii) if $H^{*}(X ; \mathrm{R}) \cong \Lambda(y)$, then

$$
H_{\mathrm{T}}^{*}(L X ; \mathrm{R}) \cong \oplus_{k \geq 0} \mathrm{R}\{\beta(k)\} \oplus \mathrm{R}[u]
$$

as an algebra, where $\operatorname{deg} \beta(k)=(k+1)(\operatorname{deg} y-1), \beta(k) * \beta(j)=\beta(k+j+1)$ and $\beta(k) * u=0$ for any $j, k$.

As for the algebra structure of $H_{\mathrm{T}}^{*}(L X ; \mathrm{R})$, the above results cover [13, Theorem 2.4].

This paper is set out as follows. In Section 1, we define the additive KTheory $K_{*}^{+}(\Omega)$ of a DGA $(\Omega, d)$ over a commutative ring and a product (*-product) on $K_{*}^{+}(\Omega)$. Some properties of the *-product will also be studied. 
In Section 2, we will describe the applications of Connes' B-maps $B$ and $B_{\mathrm{HH}}$ which are mentioned above.

\section{$\S 1$. The *-product on $K_{*}^{+}$}

Let $(\Omega, d)$ be a commutative differential graded algebra (DGA) over a commutative ring $\mathbf{k}, \Omega=\bigoplus_{i \leq 0} \Omega_{i}$, with unit 1 in $\Omega_{0}$, endowed with a differential $d$ of degree -1 satisfying $d(1)=0$. We assume that differential graded algebras are non-positively graded algebras with the above properties unless otherwise stated. We recall the cyclic bar complex defined in [7] and [8]. The complex $\left(\mathbf{C}(\Omega)\left[u^{-1}\right], b+u B\right)$ is defined as follows:

$$
\begin{gathered}
\mathbf{C}(\Omega)=\sum_{k=0}^{\infty} \Omega \otimes \bar{\Omega}^{\otimes k}, \\
b\left(\omega_{0}, . ., \omega_{k}\right)=-\sum_{i=0}^{k}(-1)^{\varepsilon_{i-1}}\left(\omega_{0}, . ., \omega_{i-1}, d \omega_{i}, \omega_{i+1}, . ., \omega_{k}\right) \\
-\sum_{i=0}^{k-1}(-1)^{\varepsilon_{i}}\left(\omega_{0}, . ., \omega_{i-1}, \omega_{i} \omega_{i+1}, \omega_{i+2}, . ., \omega_{k}\right) \\
+(-1)^{\left(\operatorname{deg} \omega_{k}-1\right) \varepsilon_{k-1}}\left(\omega_{k} \omega_{0}, . ., \omega_{k-1}\right), b\left(u^{-1}\right)=0
\end{gathered}
$$

and

$B\left(\omega_{0}, . ., \omega_{k}\right)=\sum_{i=0}^{k}(-1)\left(\varepsilon_{i-1}+1\right)\left(\varepsilon_{k}-\varepsilon_{i-1}\right)\left(1, \omega_{i}, . ., \omega_{k}, \omega_{0}, . ., \omega_{i-1}\right), \quad B\left(u^{-1}\right)=0$,

where $\bar{\Omega}=\Omega / \mathbf{k}, \operatorname{deg}\left(\omega_{0}, . ., \omega_{k}\right)=\operatorname{deg} \omega_{0}+\cdots+\operatorname{deg} \omega_{k}+k$, for $\left(\omega_{0}, . ., \omega_{k}\right)$ in $\mathbf{C}(\Omega), \varepsilon_{i}=\operatorname{deg} \omega_{0}+\cdots+\operatorname{deg} \omega_{i}-i$ and $\operatorname{deg} u=-2$. Note that the formulas $b B+B b=0$ and $b^{2}=B^{2}=0$ hold, see [7]. The negative cyclic homology $\mathrm{HC}_{*}^{-}(\Omega)$, the periodic cyclic homology $\mathrm{HC}_{*}^{\mathrm{per}}(\Omega)$ and the Hochschild homology $\mathrm{HH}_{*}(\Omega)$ of a DGA $(\Omega, d)$ are defined as the homology of the complexes $(\mathbf{C}(\Omega)[[u]], b+u B),\left(\mathbf{C}(\Omega)\left[\left[u, u^{-1}\right], b+u B\right)\right.$ and $(\mathbf{C}(\Omega), b)$, respectively. Since a DGA in our case has negative degrees, the power series algebra $\mathbf{C}(\Omega)[[u]]$ agrees with the polynomial algebra $\mathbf{C}(\Omega)[u]$, similarly, $\mathbf{C}(\Omega)\left[\left[u, u^{-1}\right]=\mathbf{C}(\Omega)\left[u, u^{-1}\right]\right.$.

We define the $n$th additive K-theory $K_{n}^{+}(\Omega, d)$ of a DGA $(\Omega, d)$ to be the $(n-1)$-th cyclic homology $\operatorname{HC}_{n-1}(\Omega, d)$ which is the $(n-1)$-th homology of the cyclic bar complex $\left(\mathbf{C}(\Omega)\left[u^{-1}\right], b+u B\right)$ :

$$
K_{*}^{+}(\Omega)=\mathrm{HC}_{*-1}(\Omega)=H_{*-1}\left(\mathbf{C}(\Omega)\left[u^{-1}\right], b+u B\right) .
$$


Unless we note the differential $d$ of a DGA in particular, $K_{n}^{+}(\Omega, d)$ will be denoted by $K_{n}^{+}(\Omega)$. We define a product (*-product) on the complex $\left(\mathbf{C}(\Omega)\left[u^{-1}\right], b+u B\right)$ as follows:

$$
\sum_{i=0}^{n} x_{i} u^{-i} * \sum_{j=0}^{m} y_{j} u^{-j}=\sum_{i=0}^{n} x_{i} \cdot B y_{0} u^{-i},
$$

where is the shuffle product on $\mathbf{C}(\Omega)$.

Proposition 1.1. (i) The *-product induces a degree +1 map of complexes $\mathbf{C}(\Omega)\left[u^{-1}\right] \otimes \mathbf{C}(\Omega)\left[u^{-1}\right] \rightarrow \mathbf{C}(\Omega)\left[u^{-1}\right]$ which is associative.

(ii) The *-product on the cyclic bar complex defines an associative graded commutative algebra structure on $K_{*}^{+}(\Omega)$.

In [7], to give an $A_{\infty}$-algebra structure to the graded k-module $\mathbf{C}(\Omega)[[u]]$, E. Getzler and J. D. S. Jones have defined a sequence of operators $B_{k}: \mathbf{C}(\Omega)^{\otimes k} \rightarrow \mathbf{C}(\Omega)$ of degree $\mathrm{k}$ and have clarified relation of $B_{k}, B_{k-1}$ and the shuffle products on $\mathbf{C}(\Omega)$. In particular, in order to prove Proposition 1.1 , we need the following formula representing the relation of the operator $B_{2}$, Connes' B-operator $B: \mathbf{C}(\Omega) \rightarrow \mathbf{C}(\Omega)$ and the shuffle products.

Lemma 1.2. ([7, Lemma 4.3]) There exists an operator $B_{2}: \mathbf{C}(\Omega)^{\otimes 2} \rightarrow$ $\mathbf{C}(\Omega)$ of rank 2 satisfying

$$
\begin{aligned}
& (-1)^{|\alpha|+1} b B_{2}(\alpha, \beta)+B(\alpha \cdot \beta)=(-1)^{|b \alpha|+1} B_{2}(b \alpha, \beta)+(B \alpha) \cdot \beta \\
& +(-1)^{|\alpha|}\left\{\alpha \cdot B \beta+(-1)^{|\alpha|+1} B_{2}(\alpha, b \beta)\right\} .
\end{aligned}
$$

The definitions of $B_{2}$ (see [7, page 280]) and $B$ enable us to deduce that, for any elements $z$ and $z^{\prime}$ in $\mathbf{C}(\Omega)$,

$$
B_{2}\left(z, B z^{\prime}\right)=B_{2}\left(B z, z^{\prime}\right)=0 .
$$

Proof of Proposition 1.1. (i) From the formulas (1.2.1) and (1.2.2), by replacing the element $\beta$ with $B \gamma$, it follows that $B(\alpha \cdot B \gamma)=B \alpha \cdot B \gamma$. For any elements $x=\sum x_{i} u^{-i}, y=\sum y_{j} u^{-j}$ and $z=\sum z_{k} u^{-k}$ in $\mathbf{C}(\Omega)\left[u^{-1}\right]$, we see that, on $\mathbf{C}(\Omega)\left[u^{-1}\right], x *(y * z)=x *\left(\sum y_{j} \cdot B z_{0}\right) u^{-j}=\sum x_{i} \cdot B\left(y_{0} \cdot B z_{0}\right) u^{-i}=$ $(x * y) * z$. We will prove that $*$-product is a map of complexes. Since the differential $b$ is a derivation under the shuffle product on $\mathbf{C}(\Omega)\left[u^{-1}\right]$, by the formula $b \circ B+B \circ b=0$, we have 


$$
\begin{aligned}
& (b+u B)(x * y)=(b+u B)\left(\sum_{i \geq 0}\left(x_{i} \cdot B y_{0}\right) u^{-i}\right) \\
& =\sum_{i \geq 0}\left(b x_{i}\right) \cdot B y_{0} u^{-i}+\sum_{i \geq 0}(-1)^{\left|x_{i}\right|} x_{i} \cdot b B y_{0} u^{-i}+\sum_{i \geq 0} B\left(x_{i} \cdot B y_{0}\right) u^{-i+1} \\
& =\sum_{i \geq 0}\left(b x_{i}\right) \cdot B y_{0} u^{-i}+\sum_{i \geq 0}(-1)^{\left|x_{i}\right|+1} x_{i} \cdot B b y_{0} u^{-i}+\sum_{i \geq 0} B x_{i+1} \cdot B y_{0} u^{-i} .
\end{aligned}
$$

On the other hand, by the formula $B \circ B=0$, we have

$$
\begin{aligned}
(b+u B) x * y & +(-1)^{|x|+1} x *(b+u B) y \\
& =\sum_{i \geq 0}\left(b x_{i}\right) \cdot B y_{0} u^{-i}+\sum_{i \geq 0} B x_{i+1} \cdot B y_{0} u^{-i} \\
& +(-1)^{|x|+1} x *\left(\sum_{j \geq 0} b y_{j} u^{-j}+\sum_{j \geq 0} B y_{j+1} u^{-j}\right)
\end{aligned}
$$

Thus we can conclude that $(b+u B)(x * y)=(b+u B) x * y+(-1)^{|x|+1} x *$ $(b+u B) y$. Note that $(-1)^{|x|}=(-1)^{\left|x_{i}\right|}$ for any $i$.

(ii) To prove that the *-product defines a graded commutative algebra structure on $K_{*}^{+}(\Omega)$, it suffices to prove that, for any elements $x=\sum x_{i} u^{-i}$ and $y=\sum y_{j} u^{-j}$ in $\operatorname{Ker}(b+u B)$, there exists an element $\omega=\sum_{k \geq 0} \omega_{k-1} u^{-k}$ such that

$$
x_{k} \cdot B y_{0}-(-1)^{(|x|+1)(|y|+1)} y_{k} \cdot B x_{0}=b \omega_{k-1}+B \omega_{k}
$$

for any $k \geq 0$. We will verify that

$$
\begin{aligned}
& \omega_{k}=(-1)^{(|y|+1)|x|}\left(\sum_{i+j=k} y_{i} \cdot x_{j}-\sum_{i+j=k+1}(-1)^{\left|y_{i}\right|} B_{2}\left(y_{i}, x_{j}\right)\right) \text { for } k \geq 0 \text { and } \\
& \omega_{-1}=(-1)^{(|x|+1)(|y|+1)} B_{2}\left(y_{0}, x_{0}\right)
\end{aligned}
$$

are factors of the required element. Since equalities $b y_{i}=-B y_{i+1}$ and $b x_{j}=-B_{j+1} x_{j+1}$ hold, it follows that, if $k \geq 0$, 


$$
\begin{aligned}
& (-1)^{(|y|+1)|x|}\left(b \omega_{k-1}+B \omega_{k}\right) \\
& =\sum_{i+j=k-1}\left\{-B y_{i+1} \cdot x_{j}+(-1)^{\left|y_{i}\right|} y_{i} \cdot\left(-B x_{j+1}\right)\right\}-\sum_{i+j=k}(-1)^{\left|y_{i}\right|} b B_{2}\left(y_{i}, x_{j}\right) \\
& +\sum_{i+j=k}\left\{B y_{i} \cdot x_{j}+(-1)^{\left|y_{i}\right|} y_{i} \cdot B x_{j}+(-1)^{\left|y_{i}\right|} b B_{2}\left(y_{i}, x_{j}\right)\right\} \\
& =-\sum_{i+j=k, i \geq 1} B y_{i} \cdot x_{j}-\sum_{i+j=k, j \geq 1}(-1)^{\left|y_{i}\right|} y_{i} \cdot B x_{j} \\
& +\sum_{i+j=k} B y_{i} \cdot x_{j}+\sum_{i+j=k}(-1)^{\left|y_{i}\right|} y_{i} \cdot B x_{j} \\
& =B y_{0} \cdot x_{k}+(-1)^{\left|y_{k}\right|} y_{k} \cdot B x_{0} \\
& =(-1)^{(|y|+1)|x|}\left(x_{k} \cdot B y_{0}-(-1)^{(|y|+1)(|x|+1)} y_{k} \cdot B x_{0}\right)
\end{aligned}
$$

from the formulas (1.2.1) and (1.2.2). We can check that equality $b \omega_{-1}+B \omega_{0}=x_{0} \cdot B y_{0}-(-1)^{(|x|+1)(|y|+1)} y_{0} \cdot B x_{0}$ holds in a similar way.

We define Connes' B-maps $B_{\mathrm{HH}}: K_{n}^{+}(\Omega) \longrightarrow \mathrm{HH}_{n}(\Omega)$ and $B: K_{n}^{+}(\Omega) \longrightarrow$ $\operatorname{HC}_{n}^{-}(\Omega)$ by $B_{\mathrm{HH}}\left(\sum_{i \geq 0} x_{i} u^{-i}\right)=B x_{0}$ and $B\left(\sum_{i \geq 0} x_{i} u^{-i}\right)=B x_{0}$. Note that the maps $B_{\mathrm{HH}}$ and $B$ are connecting maps in Connes' exact sequences ([16, Theorem 2.2.1 and Proposition 5.1.5])

$$
\cdots \rightarrow \mathrm{HH}_{n+1}(\Omega) \stackrel{I}{\longrightarrow} K_{n+2}^{+}(\Omega) \stackrel{S}{\longrightarrow} K_{n}^{+}(\Omega) \stackrel{B_{\mathrm{HH}}}{\longrightarrow} \mathrm{HH}_{n}(\Omega) \rightarrow \cdots
$$

and

$$
\cdots \rightarrow \mathrm{HC}_{n+1}^{-}(\Omega) \stackrel{\times u}{\longrightarrow} \mathrm{HC}_{n-1}^{\mathrm{per}}(\Omega) \longrightarrow K_{n}^{+}(\Omega) \stackrel{B}{\longrightarrow} \mathrm{HC}_{n}^{-}(\Omega) \rightarrow \cdots
$$

respectively.

Proof of TheOrem 0.1 . The product structure $m_{2}$ on $\mathbf{C}(\Omega)[u]$ defined by $m_{2}\left(\alpha_{1}, \alpha_{2}\right)=\alpha_{1} \cdot \alpha_{2}+(-1)^{\left|\alpha_{1}\right|+1} u B_{2}\left(\alpha_{1}, \alpha_{2}\right)$ induces the algebra structure of $\mathrm{HC}_{*}^{-}(\Omega)$. From (1.2.2), we see that the product $m_{2}$ agrees with the shuffle product if $\alpha_{1}$ or $\alpha_{2}$ belongs to the image of the operator $B: \mathbf{C}(\Omega) \rightarrow \mathbf{C}(\Omega)$. Therefore the formula $B(\alpha \cdot B \gamma)=B \alpha \cdot B \gamma$ implies that the map $B: K_{*}^{+}(\Omega) \rightarrow \mathrm{HC}_{*}^{-}(\Omega)$ is a morphism of algebras.

In study of the cyclic homology theory, it is often useful to consider the reduced theory. To prove some theorems below, we will use the reduced additive K-theory $\tilde{K}_{*}^{+}(\Omega)$ defined by $\tilde{K}_{*}^{+}(\Omega)=\operatorname{Coker}\left(\iota_{*}: K_{*}^{+}(\mathbf{k}) \rightarrow K_{*}^{+}(\Omega)\right)$, where $\iota: \mathbf{k} \rightarrow \Omega$ is the unit. The reduced additive $\mathrm{K}$-theory $\tilde{K}_{*}^{+}(\Omega)$ is a direct summand of $K_{*}^{+}(\Omega)$ because the exact sequence $0 \rightarrow \mathbf{C}(\mathbf{k})\left[u^{-1}\right] \rightarrow$ $\mathbf{C}(\Omega)\left[u^{-1}\right] \rightarrow \mathbf{C}(\bar{\Omega})\left[u^{-1}\right] \rightarrow 0$ of cyclic chain complexes is a split sequence. 
More precisely, $K_{*}^{+}(\Omega)$ is isomorphic to $\tilde{K}_{*}^{+}(\Omega) \oplus \mathbf{k}\left[u^{-1}\right]$ as a graded $\mathrm{HC}_{*}(\mathbf{k})=\mathbf{k}\left[u^{-1}\right]$-module. When one notices the direct summand $\mathbf{k}\left[u^{-1}\right]$ of $K_{*}^{+}(\Omega)$, by definition of *-product, it follows that $\mathbf{k}\left[u^{-1}\right]$ is included in the annihilator ideal of $K_{*}^{+}(\Omega)$. Therefore we can also conclude that the algebra $\left(K_{*}^{+}(\Omega), *\right)$ does not have an unit.

Let us consider a relation of the *-product on $K_{*}^{+}(\Omega)$ to the suspension $\operatorname{map} S: K_{*}^{+}(\Omega) \rightarrow K_{*-2}^{+}(\Omega)$ in Connes' exact sequence (1.1). Since the suspension map $S$ is defined by $S\left(\sum_{i \geq 0} x_{i} u^{-i}\right)=\sum_{i \geq 0} x_{i+1} u^{-i}$, it follows that $S x * y=S(x * y)$ on $\mathbf{C}(\Omega)\left[u^{-1}\right]$. From this fact and commutativity of the *-product, we have

Proposition 1.3. For any elements $\omega$ and $\eta$ in $K_{*}^{+}(\Omega)$,

$$
S \omega * \eta=S(\omega * \eta)=\omega * S \eta .
$$

For the rest of this paper, unless otherwise mentioned, we will assume that any DGA $(\Omega, d)$ is a commutative algebra over a field $\mathbf{k}$ of characteristic zero, connected and simply connected, that is, $\Omega=\oplus_{i \leq 0} \Omega_{i}, \Omega_{0}=\mathbf{k}$, $H_{1}(\Omega)=0$ and $d(1)=0$. A DGA $(\Omega, d)$ is said to be formal if there exists a DGA-morphism from the minimal model $\mathscr{M}$ of $\Omega$ to the DGA $\left(H^{*}(\Omega, d), 0\right)$ which induces a isomorphism between their homologies (see [10]).

For any DGA $(\Omega, 0)$ with the trivial differential, M. Vigué-Poirrier has given a decomposition of the negative cyclic homology $\operatorname{HC}_{*}^{-}(\Omega, 0)$ : $\mathrm{HC}_{*}^{-}(\Omega, 0)=\oplus_{q \geq 0} H\left(\mathscr{C}_{*}^{q}[u], b+u B\right)$, and has shown that the S-action on $\widetilde{\mathrm{HC}}_{*}^{-}(\Omega, 0)$ is trivial, see [22, Proposition 5], where $\mathscr{C}_{n}^{q}=\left\{\left(a_{0}, \ldots, a_{p}\right) \mid\right.$ $\left.\sum \operatorname{deg} a_{i}=-q,-q+p=-n\right\}$. This fact implies that the S-action on $\widetilde{\mathrm{HC}_{*}^{-}}(\Omega, d)$ for any formal DGA $(\Omega, d)$ is trivial ([22, Théorème $\left.\left.\mathrm{A}\right]\right)$. The proof of [22, Proposition 5] is based on Goodwillie's result [9, Corollary III.4.4], which is led from the following proposition.

Proposition 1.4. [9]. Let $(\Omega, d)$ be a DGA over a commutative ring and $D a$ derivation on $\Omega$ with degree $|D|$ satisfying that $D(a b)=(D a) b+(-1)^{|D \|| a \mid} a(D b)$ and $[D, d]=0$. Then there exist chain maps $e_{D}: \mathbf{C}(\Omega) \rightarrow \mathbf{C}(\Omega)$ of degree $|D|-1, \quad E_{D}: \mathbf{C}(\Omega) \rightarrow \mathbf{C}(\Omega) \quad$ of degree $|D|+1$ and an operator $L_{D}: \mathbf{C}(\Omega) \rightarrow \mathbf{C}(\Omega)$ of degree $|D|$ such that $\left[u^{-1} e_{D}+E_{D}, b+u B\right]=L_{D}$ in $\mathbf{C}(\Omega)\left[u, u^{-1}\right]$, where $[a, b]=a b-(-1)^{|a||b|} b a$ for any operators $a$ and $b$.

We can obtain a lemma by using Proposition 1.4 and the idea of the decomposition of cyclic homology due to Vigué-Poirrier [22].

Lemma 1.5. Let $(\Omega, 0)$ be a DGA with the trivial differential. For any ele- 
ment $\omega$ in $\tilde{K}_{*}^{+}(\Omega, 0)=\widetilde{\mathrm{HC}}_{*-1}(\Omega, 0)$, there exists an element $\eta_{0}$ in $\mathbf{C}(\Omega) \cap \operatorname{ker} b$ such that $\omega=\left[\eta_{0}\right]$ in $\tilde{K}_{*}^{+}(\Omega, 0)$.

Proof. According to Vigué-Poirrier [22], we define a derivation $D$ on $\Omega$ by $D(a)=(\operatorname{deg} a) a$. Consider a decomposition $K_{*}^{+}(\Omega, 0)=\sum_{q \geq 0} K_{*}^{+}(\Omega, 0)^{q}$ defined by $K_{*}^{+}(\Omega, 0)^{q}=H_{*-1}\left(\mathscr{C}_{*}^{q}\left[u^{-1}\right], b+u B\right)$. Since $\tilde{K}_{*}^{+}(\Omega)$ is isomorphic to $\sum_{q>1} K_{*}^{+}(\Omega)^{q}$, in order to prove Lemma 1.5 , it suffices to show that there exists an element $\eta_{0}$ with the property in Lemma 1.5 for any element $\omega$ in $K_{*}^{+}(\Omega)^{q} \quad(q \geq 1)$. Since the operation $L_{D}$ on $\mathbf{C}(\Omega)$ is defined by $L_{D}\left(a_{0}, . ., a_{p}\right)=\sum_{0 \leq i \leq p}\left(a_{0}, . ., D a_{i}, . ., a_{p}\right)$, it follows that the operator $L_{D}$ on $K_{*}^{+}(\Omega)^{q}$ is given by $L_{D}(\omega)=-q \omega$ in our case. On the other hand, for any element $\omega$ in $K_{*}^{+}(\Omega)^{q}$ which is represented by $\sum_{i>0} \omega_{i} u^{-i}$ in $\mathbf{C}(\Omega)\left[u^{-1}\right]$, we have that $\left[u^{-1} e_{D}+E_{D}, b+u B\right] \omega=e_{D} B \omega_{0}+u E_{D} B \omega_{0}-(b+u B)\left(u^{-1} e_{D}+\right.$ $\left.E_{D}\right) \omega$ in $\mathbf{C}(\Omega)\left[u, u^{-1}\right]$. By virtue of Proposition 1.4, we can conclude that $e_{D} B \omega_{0}-(b+u B)\left(u^{-1} e_{D}+E_{D}\right) \omega=-q \omega$ in $\mathbf{C}(\Omega)\left[u^{-1}\right]$. Thus, we see that $-\frac{1}{q} e_{D} B \omega_{0}$ is the required element $\eta_{0}$.

We will consider the algebra structure of $K_{*}^{+}(\Omega)$ by using a minimal model of $(\Omega, d)$. Let $\varphi:\left(\mathscr{M}, d_{\mathscr{M}}\right) \longrightarrow(\Omega, d)$ be a minimal model of a DGA $(\Omega, d)$. Then $\varphi$ induces an isomorphism of algebras $K(\varphi): K_{*}^{+}(\mathscr{M}) \longrightarrow K_{*}^{+}(\Omega)$. Therefore if a DGA $(\Omega, d)$ is formal, then there exist isomorphisms $K_{*}^{+}(\Omega, d) \cong K_{*}^{+}\left(\mathscr{M}, d_{M}\right) \cong K_{*}^{+}(H(\Omega), 0)$. It follows immediately that the isomorphisms are compatible with the S-action. Since Lemma 1.5 asserts that any element of $\tilde{K}_{*}^{+}(\Omega, 0)$ can be represented by an element with column degree 0 , from the definition of S-action, we can get

Proposition 1.6. If a DGA $(\Omega, d)$ is formal, then the suspension map $S: \tilde{K}_{*}^{+}(\Omega) \longrightarrow \tilde{K}_{*-2}^{+}(\Omega)$ is trivial.

Let $\left(\mathscr{M}, d_{\mathscr{M}}\right)$ be a free commutative differential graded algebra $(\Lambda V, d)$ over k. We denote by $(\mathscr{E}(\mathscr{M}), \delta, \beta)$ the double complex defined in $[4$, Example 2] by D. Burghelea and M. Vigué-Poirrier. Namely, $\mathscr{E}(\mathscr{M})=$ $\Lambda(V \oplus \bar{V}), \beta$ is the unique derivation of degree +1 defined by $\beta v=\bar{v}$ and $\delta$ is the unique derivation of degree -1 which satisfies $\left.\delta\right|_{V}=d$ and $\delta \beta+\beta \delta=0$. Here $\bar{V}$ is the vector space with $\bar{V}_{n+1}=V_{n}$. We here mention that the double complex induces the complex $\left(\mathscr{E}(\mathscr{M})\left[u^{-1}\right], \delta+u \beta\right)$ with a product defined by $\sum \omega_{i} u^{-i} * \sum \eta_{j} u^{-j}=\sum \omega_{i} \beta \eta_{0} u^{-i}$. By [4, Theorem 2.4 (i)], we see that the map $\Theta: \mathbf{C}(\mathscr{M}) \rightarrow \mathscr{E}(\mathscr{M})$ defined by $\Theta\left(a_{0}, a_{1}, . ., a_{p}\right)=1 / p ! a_{0} \beta a_{1} \cdots \beta a_{p}$ is a chain map between the double complexes $(\mathbf{C}(\mathscr{M}), b, B)$ and $(\mathscr{E}(\mathscr{M}), \delta, \beta)$. [4, Theorem 2.4 (iii)] shows that the induced map $K(\Theta)$ from $K_{*}^{+}(\mathscr{M})$ to $H_{*-1}\left(\mathscr{E}(\mathscr{M})\left[u^{-1}\right], \delta+u \beta\right)$ is an isomorphism of graded vector spaces. Moreover we have 
Proposition 1.7. The map $K(\Theta): K_{*}^{+}(\mathscr{M}) \rightarrow H_{*-1}\left(\mathscr{E}(\mathscr{M})\left[u^{-1}\right], \delta+u \beta\right)$ is an isomorphism of algebras.

The following lemma will be needed to prove that $K(\Theta)$ is a morphism of algebras.

LeMma 1.8. Let $\left(\mathscr{M}, d_{\mathscr{M}}\right)$ be a free commutative DGA.

(i) The chain map $\Theta: \mathbf{C}(\mathscr{M}) \rightarrow \mathscr{E}(\mathscr{M})$ is compatible with $B: \mathbf{C}(\Omega) \rightarrow \mathbf{C}(\Omega)$ and $\beta: \mathscr{E}(\mathscr{M}) \rightarrow \mathscr{E}(\mathscr{M}): \beta \Theta=\Theta B$.

(ii) Let $W$ be a subspace of $\mathbf{C}(\mathscr{M})$ consisting of the elements whose first factor have even degree: $W=\left\{\sum_{i}\left(a_{i_{0}}, . ., a_{i_{k}(i)}\right) \in \mathbf{C}(\mathscr{M}) \mid \operatorname{deg} a_{i_{0}}\right.$ is even $\}$. Then $\Theta\left(\omega \cdot \omega^{\prime}\right)=\Theta \omega \cdot \Theta \omega^{\prime}$ for any element $\omega^{\prime}$ in $W$ and any element $\omega$ in $\mathbf{C}(\mathscr{M})$, here in the left hand side and right hand side are the shuffle product on $\mathbf{C}(\mathscr{M})$ and the natural product on $\mathscr{E}(\mathscr{M})$ respectively.

Proof. It is straightforward to check that identities $\beta \Theta=\Theta B$ and $\Theta\left(\omega \cdot \omega^{\prime}\right)=\Theta \omega \cdot \Theta \omega^{\prime}$ hold.

Proof of Proposition 1.7. From the definition of Connes' B-operator, it follows that $\operatorname{Im} B$ is a subspace of $W$ in Lemma 1.8. By virtue of Lemma 1.8, we see that $\Theta\left(\omega \cdot B \omega^{\prime}\right)=\Theta \omega \cdot \beta \Theta \omega^{\prime}$ for any element $\omega$ and $\omega^{\prime}$ in $\mathbf{C}(\mathscr{M})$. Thus we can conclude that $K(\Theta)$ is a morphism of graded algebras.

By virtue of Proposition 1.7, we can determine $K_{*}^{+}(\Omega)$ explicitly as an algebra when the homology of $(\Omega, d)$ is generated with one generator.

THEOREM 1.9. For any formal DGA $(\Omega, d)$,

$$
\begin{aligned}
K_{*}^{+}(\Omega) & \cong \widetilde{\mathrm{HH}}_{*-1}(\Omega) / \operatorname{Im}\left(B_{\mathrm{HH}} \circ I: \widetilde{\mathrm{HH}}_{*-2}(\Omega)\right. \\
& \left.\rightarrow \widetilde{\mathrm{HH}}_{*-1}(\Omega)\right) \oplus \mathbf{k}\left\{1, u^{-1}, u^{-2}, . .\right\}
\end{aligned}
$$

as an algebra, where $\operatorname{deg} u^{-k}=2 k+1, \omega * \omega^{\prime}=\omega \cdot B \omega^{\prime}, \omega * u^{-k}=0$ for any elements $\omega$ and $\omega^{\prime}$ in $\widehat{\mathrm{HH}}_{*}(\Omega) / \operatorname{Im}\left(B_{\mathrm{HH}} \circ I\right)$ and $u^{-i} * u^{-j}=0$. In particular,

(i) when deg $x$ is even,

$$
K_{*}^{+}\left(\mathbf{k}[x] /\left(x^{s+1}\right)\right) \cong \oplus_{k \geq 0,1 \leq j \leq s} \mathbf{k}\{\beta(j, k)\} \oplus \mathbf{k}\left\{1, u^{-1}, u^{-2}, . .\right\},
$$

where $\operatorname{deg} \beta(j, k)=j \operatorname{deg} x+k((s+1) \operatorname{deg} x+2)+1, \omega * \omega^{\prime}=0$ for any elements $\omega$ and $\omega^{\prime}$ in $K_{*}^{+}\left(\mathbf{k}[x] /\left(x^{s+1}\right)\right)$, and

(ii) when $\operatorname{deg} y$ is odd,

$$
K_{*}^{+}(\Lambda(y)) \cong \oplus_{k \geq 0} \mathbf{k}\{\beta(k)\} \oplus \mathbf{k}\left\{1, u^{-1}, u^{-2}, . .\right\}
$$

where $\operatorname{deg} \beta(k)=\operatorname{deg} y+k(\operatorname{deg} y+1)+1, \beta(k) * \beta(j)=\beta(k+j+1), 1 * \beta(k)=0$ and $\beta(k) * u^{-l}=0$.

Proof. By Proposition 1.6, the suspension map $S: \tilde{K}_{*}^{+}(\Omega) \rightarrow \tilde{K}_{*-2}^{+}(\Omega)$ is 
trivial. From this fact and Connes' exact sequence (1.1) obtained by using $\bar{\Omega}$ instead of a DGA $\Omega$, it follows that the map $I: \widetilde{\mathrm{HH}}_{*-1}(\Omega) \rightarrow \tilde{K}_{*}^{+}(\Omega)$ is surjective and that the kernel of $I$ is the image of $B_{\mathrm{HH}} \circ I$ : $\widetilde{\mathrm{HH}}_{*-2}(\Omega) \rightarrow \widehat{\mathrm{HH}}_{*-1}(\Omega)$. Thus we can conclude that $K_{*}^{+}(\Omega) \cong \tilde{K}_{*}^{+}(\Omega) \oplus$ $\mathbf{k}\left\{1, u^{-1}, u^{-2}, ..\right\} \cong \widehat{\mathrm{HH}}_{*-1}(\Omega) / \operatorname{Im}\left(B_{\mathrm{HH}} \circ I\right) \oplus \mathbf{k}\left\{1, u^{-1}, u^{-2}, ..\right\}$ as algebras. From Proposition 1.7 and the explicit formulas of the Hochschild homology of $\mathbf{k}[x] /\left(x^{s+1}\right)$ and $\Lambda(y)$ in [15], we can get (i) and (ii).

REMARK. In Theorem 1.9 , the elements $\beta(j, k)$ and $\beta(k)$ correspond to the elements $x^{j} \omega^{k}$ and $y \bar{y}^{k}$ in [15, Proposition 1.1(ii)], respectively.

As mentioned before Proposition 1.3, the algebra $K_{*}^{+}(\Omega)$ does not have an unit. Since $\tilde{K}_{0}^{+}(\Omega)$ is non zero in general, the algebra $\tilde{K}_{*}^{+}(\Omega)$ may be have an unit. However, the results of Theorem 1.9 (i) and (ii) enable us to conjecture that the reduced additive $\mathrm{K}$-theory $\tilde{K}_{*}^{+}$does not have an unit for any DGA either. The first assertion in the following proposition is an answer to the conjecture.

Proposition 1.10. (i) Let $(\Omega, d)$ be a DGA. Assume that $\tilde{K}_{*}^{+}(\Omega) \neq 0$. Then the algebra $\tilde{K}_{*}^{+}(\Omega)$ does not have an unit.

(ii) If $\operatorname{deg} Q H(\Omega, d) \geq n$, then there exist $n$ elements $x_{1}, x_{2}, \ldots, x_{n}$ in $K_{*}^{+}(\Omega)$ such that $x_{1} * x_{2} * \cdots * x_{n} \neq 0$, where $Q H(\Omega, d)$ denotes the space of indecomposable elements in the graded algebra $H(\Omega, d)$.

Proof. From the usual argument on a minimal model of $\Omega$, we can assume that $\Omega$ is free.

(i) Suppose that there exists an element $e$ in $\tilde{K}_{*}^{+}(\Omega)$ such that $e * x=x$ for any $x$ in $\tilde{K}_{*}^{+}(\Omega)$. Let us consider the Hodge decomposition of Hochschild homology ([3], [4, Theorem 3.1]): ${\widetilde{\mathrm{HH}_{*}}}_{*}(\Omega)=\oplus_{i \geq 0} \widetilde{\mathrm{HH}}_{*}^{(i)}(\Omega)$. Since $B_{\mathrm{HH}}: \tilde{K}_{*}^{+}(\Omega) \rightarrow \widetilde{\mathrm{HH}}_{*}(\Omega)$ is a morphism of algebras by Theorem 0.1 , it follows that $B_{\mathrm{HH}}(e) \cdot B_{\mathrm{HH}}(x)=B_{\mathrm{HH}}(x)$. We see that $B_{\mathrm{HH}}(e)$ belongs to $\mathrm{HH}_{*}^{(0)}(\Omega)$ because deg $B_{\mathrm{HH}}(e)=0$. The definition of the Hodge decomposition and Lemma 1.8 (i) enables us to deduce that $\operatorname{Im~} B_{\mathrm{HH}}$ is included in $\oplus_{i \geq 1} \widetilde{\mathrm{HH}}_{*}^{(i)}(\Omega)$. Thus we have $B_{\mathrm{HH}}(e)=0$. On the other hand, we see that $S^{N} e=0$ for some sufficient large integer $N$. If $B_{\mathrm{HH}}(x)=0$ for all $x \in \tilde{K}_{*}^{+}(\Omega)$, then the map $S: \tilde{K}_{*+2}^{+}(\Omega) \rightarrow \tilde{K}_{*}^{+}(\Omega)$ is epimorphism. Therefore, for any $x \in \tilde{K}_{*}^{+}(\Omega)$, there is an element $x^{\prime} \in \tilde{K}_{*}^{+}(\Omega)$ such that $S^{N} x^{\prime}=x$. It follows from Proposition 1.3 that $x=e * x=e * S^{N} x^{\prime}=S^{N} e * x^{\prime}=0$ for any $x$, which a contradiction. Thus $B_{\mathrm{HH}}(x) \neq 0$ for some $x \in \tilde{K}_{*}^{+}(\Omega)$. However, $B_{\mathrm{HH}}(x)=B_{\mathrm{HH}}(e) \cdot B_{\mathrm{HH}}(x)=0$. The result now follows.

(ii) We can choose $n$ elements of $\Omega$ corresponding to $x_{i}$ in $K_{*}^{+}(\Omega)$ which are part of generators of $\Omega$. We represent the elements with the same notation $x_{1}, \ldots, x_{n}$, respectively. Under the isomorphism $H(\Theta)$ in $[4$, Theorem 2.4 
(ii)], $B_{\mathrm{HH}}\left(x_{1} * \cdots * x_{n}\right)=B_{\mathrm{HH}} x_{1} \cdots B_{\mathrm{HH}} x_{n}=\bar{x}_{1} \cdots \bar{x}_{n}$ in $H_{*}(\operatorname{Tot} \mathscr{E}(\Omega), \delta)$. Since $\operatorname{Im} \delta$ consists of elements whose factors have an element in $\Omega$, it follows that $\bar{x}_{1} \cdots \bar{x}_{n} \neq 0$ in $\mathrm{HH}_{*}(\Omega) \cong H_{*}$ (Tot $\left.\mathscr{E}(\Omega), \delta\right)$. By virtue of Proposition 1.7 , we can see that $x_{1} * \cdots * x_{n} \neq 0$ in $K_{*}^{+}(\Omega)$.

From Proposition 1.10 (ii), Theorem 1.9 (i) and (ii), we can conclude that $K_{*}^{+}(\Omega)$ has trivial algebra structure if and only if the homology of $(\Omega, d)$ is generated by one element with even degree.

\section{§2. Applications of Connes' B-maps $B_{\mathrm{HH}}$ and $B$}

Let $M$ be a simply connected manifold and $L M$ the space of $C^{\infty}$-free loops on $M$. When an $\mathrm{SO}(n)$-bundle $P \rightarrow M$ over $M$ has a spin structure $Q \rightarrow M$, the string class $\mu(Q)$, which belongs to $H^{3}(L M ; Z)$, is defined as an obstruction to lift the structure group $L \operatorname{Spin}(n)$ of $L Q \rightarrow L M$ to $\widehat{L \operatorname{Spin}}(n)$, for details see [18]. Here $\widehat{L \operatorname{Spin}}(n)$ is the universal central extension of $L \operatorname{Spin}(n)$ by the circle. One of important properties for the string class $\mu(Q)$ is the fact that the class $\mu(Q)$ is the image of $\frac{1}{2} p_{1}$ by the map $\int_{S^{1}} \circ \mathrm{ev}^{*}: H^{*}(M ; Z) \rightarrow$ $H^{*}\left(L M \times S^{1} ; \mathbf{Z}\right) \rightarrow H^{*-1}(L M ; \mathbf{Z})$, where $p_{1}$ is the first Pontrjagin class of the bundle $P \rightarrow M$, ev : $L M \times S^{1} \rightarrow M$ is the evaluation map and $\int_{S^{1}}$ is the integration along $S^{1}$. Let $G$ be a linear Lie group and $\xi: Q \rightarrow M$ a G-bundle over $M$. Let $\mathrm{Ch}^{p+1}(\xi)$ be the Chern character of the bundle $\xi$. The higher string classes $\tilde{C}^{p}(L \xi)(p \geq 1)$ (see [2]) in $H^{2 p+1}(L M ; \mathrm{C})$ defined for the $L G$ bundle $L \xi: L Q \rightarrow L M$ has a similar property to the ordinary string class $\mu(Q)$. Indeed, the pth string class $\tilde{C}^{p}(L \xi)$ is the image of $-(2 \pi \sqrt{-1})^{p+1} p ! C h^{p+1}(\xi)$ by the map $\int_{S^{1}} \circ \mathrm{ev}^{*}$. As mentioned in the introduction, in the study of the problem of whether the map $\int_{S^{1}} \circ \mathrm{ev}^{*}$ is injective, the Connes' B-map $B_{\mathrm{HH}}: K_{*}^{+}(\Omega(M)) \rightarrow \mathrm{HH}_{*}(\Omega(M)) \cong H^{*}(L M ; \mathrm{R})$ plays an important role. We will have the following theorem which is a generalization of [14, Theorem 2]. We may call a simply connected manifold formal if its de Rham complex is formal (see [10]).

THEOREM 2.1. Let $M$ be a simply connected manifold and formal.

(i) For any $S O(n)$-bundle $P \rightarrow M$ with a spin structure $Q \rightarrow M$, if $H^{3}(M ; Z)$ is torsion free, then the string class $\mu(Q)$ vanishes if and only if $\frac{1}{2} p_{1}$ vanishes.

(ii) Let $G$ be a linear Lie algebra and $\xi: Q \rightarrow M a G$-bundle. The string class $\tilde{C}^{p}(L \xi)$ vanishes if and only if the Chern character $\mathrm{Ch}^{p+1}(\xi)$ of the bundle $\xi$ vanishes.

By virtue of [14, Proposition 2.1], we can regard the map $\int_{S^{1}} \circ \mathrm{ev}^{*}$ : $H^{*}(M ; \mathrm{R}) \rightarrow H^{*-1}(L X ; \mathrm{R})$ as the map $\alpha: H^{*}(M ; \mathrm{R}) \rightarrow \mathrm{HH}_{-*}(\Omega(M), d)$ defined by $\alpha(x)=(1, x)$ under the identification by the iterated integral map $\sigma: \mathrm{HH}_{-*}(\Omega(M)) \rightarrow H^{*}(L M ; \mathrm{R})$ ([8]), where $\Omega_{-i}(M)$ is the $i$ th de Rham 
complex $\Omega_{\mathrm{deRham}}^{i}(M)$ and the differential $d: \Omega_{-i}(M) \rightarrow \Omega_{-i-1}(M)$ is the exterior differential on the de Rham complex $\Omega_{\mathrm{deRham}}^{*}(M)$. Thus, to prove Theorem 2.1, it suffices to show that the map $\alpha$ is injective when $M$ is formal. Note that, for any DGA $(\Omega, d)$, we can define the map $\alpha: H_{*}(\Omega) \rightarrow \mathrm{HH}_{*}(\Omega)$ by $\alpha(x)=(1, x)$. The definition of the map $\alpha$ allows us to deduce that $\alpha$ factors through Connes' B-map $B_{\mathrm{HH}}$ as follows: $\alpha=B_{\mathrm{HH}} \circ I \circ i$, where $i: H_{*}(\Omega) \rightarrow \operatorname{HH}_{*}(\Omega)$ and $I: \operatorname{HH}_{*}(\Omega) \rightarrow K_{*}^{+}(\Omega)$ are the homomorphisms induced by the natural inclusions $\Omega \rightarrow \mathbf{C}(\Omega)$ and $\mathbf{C}(\Omega) \rightarrow \mathbf{C}(\Omega)[u-1]$ respectively. For any DGA $(\Omega, d)$, we have

Lemma 2.2. The map $H_{-*}(\Omega) \stackrel{i}{\longrightarrow} \operatorname{HH}_{-*}(\Omega) \stackrel{I}{\longrightarrow} K_{-*+1}^{+}(\Omega)$ is injective.

Proof. It suffices to prove that Lemma 2.2 holds when $\Omega$ is free. In this case, we can identify $K_{*}^{+}(\Omega)$ with the homology of the complex $\left(\mathscr{E}(\Omega)\left[u^{-1}\right], \delta+u \beta\right)$ by Proposition 1.7. Since $\operatorname{Im}(\delta+u \beta) \cap \Omega$ is contained in $\operatorname{Im} d$ which is a subspace of $\Omega$, it follows that if $\operatorname{Ii}(x)$ is zero in $K_{*}^{+}(\Omega)$, then so is $x$ in $H_{*}(\Omega)$.

Proof of Theorem 2.1. The reduced additive K-theory $\tilde{K}_{*}^{+}(\Omega)$ includes $\operatorname{Im}\left(I \circ i: H_{*-1}(\Omega) \rightarrow K_{*}^{+}(\Omega)\right)$ for $*<1$. By Proposition 1.6, Connes' B-map $B_{\mathrm{HH}}: \tilde{K}_{*}^{+}(\Omega) \rightarrow \widehat{\mathrm{HH}}_{*}(\Omega)$ is injective. Therefore we can have Theorem 2.1 by virtue of Lemma 2.2 .

In general case, we can show that $\operatorname{Ii}(\operatorname{Ker} \alpha)\left(=\operatorname{Im}(I \circ i) \cap \operatorname{Ker} B_{\mathrm{HH}}\right)$ is contained in the space of annihilators of $K_{*}^{+}(\Omega)$.

Proposition 2.3. For any DGA $(\Omega, d), K_{*}^{+}(\Omega) *\left\{\operatorname{Im}(I \circ i) \cap \operatorname{Ker} B_{\mathrm{HH}}\right\}=0$.

Proof. For any element $\omega$ in $\operatorname{Im}(I \circ i) \cap \operatorname{Ker} B_{\mathrm{HH}}$, we can write $\beta \omega=\delta \eta$ for some element $\eta$ in $\mathscr{E}(\Omega)$. For any element $\omega^{\prime}$ in $\operatorname{Ker}(u \beta+\delta)$ which is the subspace of $\mathscr{E}(\Omega)\left[u^{-1}\right]$,

$$
\begin{aligned}
(u \beta+\delta)\left(\omega^{\prime} \cdot \eta\right) & =(-1)^{\operatorname{deg} \omega^{\prime}} \omega^{\prime} \cdot(u \beta+\delta) \eta \\
& =(-1)^{\operatorname{deg} \omega^{\prime}} \omega^{\prime} \cdot(0+\beta \omega) \\
& =(-1)^{\operatorname{deg} \omega^{\prime}} \omega^{\prime} * \omega
\end{aligned}
$$

Note that $\beta \eta=0$ in $\mathscr{E}(\Omega)\left[u^{-1}\right]$. Thus we see that $\omega^{\prime} * \omega=0$ in $K_{*}^{+}(\Omega)$.

We will describe some applications of Connes' B-map $B: K_{*}^{+}(\Omega) \rightarrow$ $\mathrm{HC}_{*}^{-}(\Omega)$.

Proposition 2.4. The following diagram is commutative: 


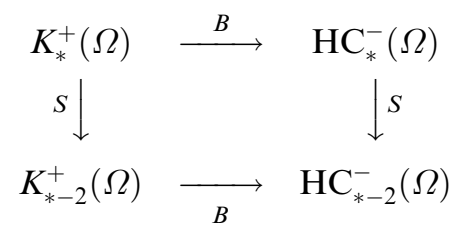

Proof. For any element $\omega=\sum \omega_{i} u^{-i}$ in $\operatorname{Ker}(b+u B)$, by the definition of the S-action, we have that $B S \omega=B \omega_{1}$. On the other hand, $S B \omega=B \omega_{0} u$. Since $b \omega_{0}+B \omega_{1}=0$, it follows that $B w_{0} u-B \omega_{1}=(b+u B) \omega_{0}$. Thus we have $S B \omega=B S \omega$ in $\mathrm{HC}_{*}^{-}(\Omega)$.

If the S-action on $\widetilde{\mathrm{HC}}_{*}^{-}(\Omega)$ is trivial, then we can represent the algebra structure of the negative cyclic homology $\operatorname{HC}_{*}^{-}(\Omega)$ with the *-product on $K_{*}^{+}(\Omega)$.

THEOREM 2.5. (i) The map B: $\tilde{K}_{*}^{+}(\Omega) \longrightarrow \widetilde{\mathrm{HC}}_{*}^{-}(\Omega)$ induced by Connes' $B$ map is an isomorphism of algebras.

(ii) The $S$-action on $\tilde{K}_{*}^{+}(\Omega)$ is trivial if and only if so is the S-action on $\widetilde{\mathrm{HC}_{*}^{-}}(\Omega)$.

(iii) If the S-action on $\widetilde{\mathrm{HC}}_{*}^{-}(\Omega)$ is trivial, then $\mathrm{HC}_{*}^{-}(\Omega) \cong \mathbf{k}[u] \oplus \tilde{K}_{*}^{+}(\Omega) \cong$ $\mathbf{k}[u] \oplus \widetilde{\mathrm{HH}}_{*-1}(\Omega) / \operatorname{Im}\left(B_{\mathrm{HH}} \circ I\right)$ as algebras. By the assertions (i) and (ii), we see that $\mathbf{k}[u] \oplus \widehat{\mathrm{HC}}_{*}^{-}(\Omega) \cong \mathbf{k}[u] \oplus \tilde{K}_{*}^{+}(\Omega)$ as an algebra.

Proof. (i) The result [9, Theorem III.5.1] enables us to conclude that $\operatorname{HC}^{\text {per }}(\Omega) \cong \mathbf{k}\left[u, u^{-1}\right]$. From Connes' exact sequence (1.2) for $\bar{\Omega}$, we can get (i). From (i) and Proposition 2.4, we have (ii). Since the S-action on $\mathrm{HC}_{*}^{-}(\Omega)$ is trivial, it follows that $\operatorname{HC}_{*}^{-}(\Omega) \cong \mathbf{k}[u] \oplus \widehat{H C}_{*}^{-}(\Omega)$ as an algebra. From the proof of Theorem 1.9, we deduce the results of (iii).

We can now prove Theorem 0.2 .

Proof of Theorem 0.2 . If $H^{*}(X ; \mathrm{R})$ is isomorphic to the algebra $\mathrm{R}[x] /\left(x^{s+1}\right)$ or $\Lambda(y)$, then $X$ is a formal. By virtue of Theorem 2.5 (iii), we see that $H_{T}^{*}(L X ; \mathrm{R}) \cong \mathrm{HC}_{-*}^{-}(\Omega(X)) \cong \mathrm{HC}_{-*}^{-}\left(H^{*}(X ; \mathrm{R})\right) \cong \mathrm{R}[u] \oplus \tilde{K}_{-*}^{+}\left(H^{*}(X ; \mathrm{R})\right)$. Therefore, Theorem 1.9 yields Theorem 0.2 . In particular, we deduce (i) and (ii) by virtue of Theorem 1.9 (i) and (ii).

Let $M$ be a simply connected manifold (simplicial complex) and $\Omega^{*}(M)$ its de Rham algebra of differential forms (simplicial differential forms) with coefficient in $\mathbf{k}=\mathrm{R}, \mathrm{C}(\mathbf{k}=\mathrm{R}, \mathrm{C}, \mathrm{Q})$. Then the isomorphism $B: \tilde{K}_{*}^{+}(\Omega) \rightarrow$ $\widetilde{\mathrm{HC}}_{*}^{-}(\Omega)$ in Theorem 2.5 (i) agrees with the isomorphism $b_{M}: \widehat{\mathrm{HC}}_{-*-1}(\Omega(M)) \rightarrow \tilde{H}_{\mathrm{T}}^{*}(L M ; \mathbf{k})$ in [3, Theorem B]. Therefore, if we regard $\tilde{K}_{*}^{+}(\Omega)$ as a graded algebra with the *-product, the isomorphism $b_{M}$ becomes a morphism of algebras. 
Let $(\Omega, d)$ and $\left(\Omega^{\prime}, d^{\prime}\right)$ be DGAs over a field $\mathbf{k}$ of characteristic zero. If one wants to know about the k-module structure of the negative cyclic homology $\mathrm{HC}_{*}^{-}\left(\Omega \otimes \Omega^{\prime}\right)$, the use of the Künneth theorem [11, Theorem 3.1 (a)] for negative cyclic homology theory may be effective, because the exact sequence

$$
\begin{aligned}
& 0 \rightarrow \operatorname{HC}_{*}^{-}(\Omega) \otimes_{\mathbf{k}[u]} \operatorname{HC}_{*}^{-}\left(\Omega^{\prime}\right) \rightarrow \operatorname{HC}_{*}^{-}\left(\Omega \otimes \Omega^{\prime}\right) \rightarrow \\
& \operatorname{Tor}_{\mathbf{k}[u]}\left(\operatorname{HC}_{*}^{-}(\Omega), \operatorname{HC}_{*}^{-}\left(\Omega^{\prime}\right)\right)_{*-1} \rightarrow 0
\end{aligned}
$$

is split. However, it is not easy to determine the algebra structure of $\mathrm{HC}_{*}^{-}\left(\Omega \otimes \Omega^{\prime}\right)$ from the exact sequence even if $\Omega$ and $\Omega^{\prime}$ are formal. Theorem 2.5 (ii) enables us to represent the graded algebra structure of $\operatorname{HC}_{*}^{-}\left(\Omega \otimes \Omega^{\prime}\right)$ with the Hochschild homologies $\operatorname{HH}_{*}(\Omega), \operatorname{HH}_{*}\left(\Omega^{\prime}\right)$ and the *-product when $\Omega$ and $\Omega^{\prime}$ are formal. In term of spaces, we also assert that the T-equivariant cohomology of the space of loops on the product space $M \times M^{\prime}$ can be represented with the cohomologies of the spaces of loops on $M$ and $M^{\prime}$, Connes' B-map $B_{\mathrm{HH}}$ and *-product.

Corollary 2.6. Let $M$ and $M^{\prime}$ be formal simply connected manifolds. Then

$$
\begin{aligned}
& H_{\mathrm{T}}^{*}\left(L\left(M \times M^{\prime}\right) ; \mathrm{R}\right) \cong \\
& \left\{\left(H^{*}(L M ; \mathrm{R}) \otimes H^{*}\left(L M^{\prime} ; \mathrm{R}\right) / \operatorname{Im}(B \circ I \otimes 1 \pm 1 \otimes B \circ I)\right)\right\}^{*+1} \oplus \mathrm{R}[u]
\end{aligned}
$$

as an algebra, where $\operatorname{deg} u=2$. Here the multiplication $*$ of the algebra on the right hand side is given as follows: $\omega \otimes \omega^{\prime} * u=0, \quad \omega \otimes \omega^{\prime} * \eta \otimes \eta^{\prime}=$ $\omega \otimes \omega^{\prime} \cdot\left(B I \eta \otimes \eta^{\prime} \quad+(-1)^{|\eta|} \eta \otimes B I \eta^{\prime}\right) \quad$ for $\quad$ any $\quad \omega \otimes \omega^{\prime} \quad$ and $\eta \otimes \eta^{\prime} \quad$ in $H^{*}(L M ; \mathrm{R}) \otimes H^{*}\left(L M^{\prime} ; \mathrm{R}\right) / \operatorname{Im}(B \circ I \otimes 1 \pm 1 \otimes B \circ I)$, where $\cdot$ is the cup product on $H^{*}(L M ; \mathrm{R}) \otimes H^{*}\left(L M^{\prime} ; \mathrm{R}\right)$.

Proof. Let $(\mathscr{M}, d)$ and $\left(\mathscr{M}^{\prime}, d\right)$ be minimal models of de Rham complexes $(\Omega(M), d)$ and $\left(\Omega\left(M^{\prime}\right), d\right)$ respectively. We know that $\mathrm{HH}_{-*}(\mathscr{M}) \cong$ $H^{*}(L M ; \mathrm{R})$ and $\mathrm{HC}_{-*}^{-}(\mathscr{M}) \cong H_{\mathrm{T}}^{*}(L M ; \mathrm{R})$ as algebras $([8])$. By virtue of [22, Proposition 5], the S-action on $\mathrm{HC}_{-*}^{-}(\mathscr{M})$ is trivial. Therefore, it follows from Theorem 2.5 (ii) that, as algebras, 


$$
\begin{aligned}
H_{\mathrm{T}}^{*}\left(L\left(M \times M^{\prime}\right) ; \mathrm{R}\right) \cong & \mathrm{HC}_{-*}^{-}\left(\mathscr{M} \otimes \mathscr{M}^{\prime}\right) \\
\cong & \mathrm{HH}_{-*-1}(\mathscr{M} \otimes \mathscr{M}) / \mathrm{Im}\left(B_{\mathrm{HH}} \circ I\right) \oplus \mathrm{R}[u] \\
\cong & H_{-*-1}\left(\mathscr{E}\left(\mathscr{M} \otimes \mathscr{M}^{\prime}\right)\right) / \operatorname{Im}\left(B_{\mathrm{HH}} \circ I\right) \oplus \mathrm{R}[u] \\
\cong & \left\{H _ { * } \left(\mathscr{E}(\mathscr{M}) \otimes H_{*}\left(\mathscr{E}\left(\mathscr{M}^{\prime}\right)\right) /\right.\right. \\
& \operatorname{Im}(\beta \circ I \otimes 1 \pm 1 \otimes \beta \circ I)\}_{-*-1} \oplus \mathrm{R}[u] \\
\cong & \left\{H^{*}(L M ; \mathrm{R}) \otimes H^{*}\left(L M^{\prime} ; \mathrm{R}\right) /\right. \\
& \operatorname{Im}(B \circ I \otimes 1 \pm 1 \otimes B \circ I)\}^{*+1} \oplus \mathrm{R}[u] .
\end{aligned}
$$

\section{REFERENCES}

1. W. Andrzejewski and A. Tralle, Cohomology of some graded differential algebras, Fund. Math. 145 (1994), 181-204.

2. A. Asada, Four Lectures on Geometry of Loop Group and Non Abelian de Rham Theory, Chalmers University of Technology/The University of Göteborg, 1990.

3. D. Burghelea, Z. Fiedorowicz and W. Gajda, Adams operations in Hochschild and cyclic homology of de Rham algebra and free loop spaces, K-theory 4 (1991) 269-287.

4. D. Burghelea and M. Vigué-Poirrier, Cyclic Homology of Commutative Algebras I, Lecture Notes in Math. 1318, 51-72.

5. A. Connes, Non commutative differential geometry Part I, II, Publ. Math. Inst. Hautes Etud. Soc. 62 (1985), 41-93, 94-144.

6. B. L. Feigin and B. L. Tsygan, Additive K-theory, Lecture Notes in Math. 1289, 67-209.

7. E. Getzler and J. D. S. Jones, $A_{\infty}$ - algebras and cyclic bar complex, Illinois J. Math. 34 (1990), 256-283.

8. E. Getzler, J. D. S. Jones and S. Petrack, Differential form on loop spaces and the cyclic bar complex, Topology 30 (1991), 339-371.

9. T. G. Goodwillie, Cyclic homology, derivations and the free loop space, Topology 24 (1985), $187-215$.

10. P. Griffiths and J.Morgan, Rational homotopy theory and differential forms, Prog. Math. 16 (1981), Birkhäuser.

11. C. Hood and J. D. S. Jones, Some algebraic properties of cyclic homology groups, K-theory 1 (1987), 361-384.

12. M. Karoubi, Homologie cyclique et K-theorie algébrique, I et II, C. R. Acad. Sci. Paris 297 (1983), 447-450, 513-516.

13. K. Kuribayashi, On the real cohomology of spaces of free loops on manifolds, Fund. Math. 150 (1996), 173-188.

14. K. Kuribayashi, On the vanishing problem of string classes, J. Austral. Math. Soc. Ser. A 61 (1996), 258-266.

15. K. Kuribayashi and T. Yamaguchi, The cohomology algebra of certain free loop spaces, Fund. Math. 154 (1997), 57-73.

16. J. L. Loday, Cyclic homology, Grundlehren Math. Wiss. 301 (1992).

17. J. L. Loday and D. Quillen, Cyclic homology and the Lie algebra homology of matrices, Comment. Math. Helv. 59 (1984), 565-591.

18. D. A. McLaughlin, Orientation and string structures on loop space, Pacific J. Math. 155 (1992), 143-156.

19. J. Rosenberg, Algebraic K-theory and its applications, Graduate Texts in Math. 147, 1994. 
20. U. Tillmann, Relation of the van Est spectral sequence to K-theory and cyclic homology, Illinois J. Math. 37 (1993), 589-608.

21. B. L. Tsygan, Homology of matrix algebras over rings and the Hochschild homology (in Russian), Uspekhi Mat. Nauk. 38 (1983), 217-218.

22. M. Vigué-Poirrier, Homologie cyclique des espaces formels, J. Pure Appl. Algebra 91 (1994), 347-354.

DEPARTMENT OF APPLIED MATHEMATICS OKAYAMA UNIVERSITY OF SCIENCE

1-1 RIDAI-CHO

OKAYAMA 700-0005

JAPAN
DEPARTMENT OF MATHEMATICS

COLLEGE OF SCIENCE

RYUKYU UNIVERSITY

NISHIHARA-CHO

OKINAWA 903-0213

JAPAN 